\title{
Evaluación de interacción medicamentosa de voriconazol-ciclosporina en pacientes pediátricos que reciben trasplante de precursores hematopoyéticos (2013-2014)
}

\author{
Romina Valenzuela, Juan P. Torres, Carolina Salas, Iván Gajardo, Julia Palma, \\ Paula Catalán, M. Elena Santolaya y Jorge Morales
}

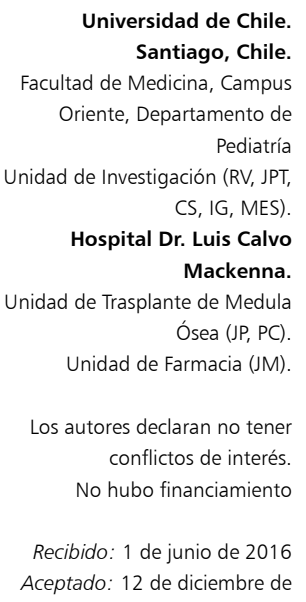

2016

Correspondencia a: Romina Valenzuela Cortés romina.v.cortes@gmail.com

\section{Drug interaction of voriconazole-cyclosporine in children undergoing hematopoietic stem cell transplantation (2013-2014)}

Background: Drug interactions (DI) in patients receiving hematopoietic stem cell transplantation (HSCT) are common and clinically significant, highlighting: anticonvulsants, voriconazole (VCZ) and cyclosporine (CsA), which require monitoring. Objective: To describe the interactions between CsA-VCZ in children undergoing HSCT. Methods: Retrospective, descriptive study in immunocompromised children hospitalized since January 2013 to December 2014 at Bone Marrow Transplant Unit, Hospital Dr. Luis Calvo Mackenna, who received CsA and VCZ. Results: The median age was 5 years (3-6) and the median weight was $20 \mathrm{~kg}$ (17-30). Sixtythree baseline drug levels were analyzed, of those, 27 were CsA drug levels obtained previous to using VCZ and 36 were CsA drug levels collected concomitantly with VCZ. In the group CsA previous to VCZ, the CsA dose was $4.6 \pm 2.6$ (mg/ kg/ day) and the CsA average level was $188.8 \pm 84.1(\mu \mathrm{g} / \mathrm{ml})$. In the group of CsA concomitantly with VCZ, the dose of CsA was $5.5 \pm 3.0(\mathrm{mg} / \mathrm{kg} /$ day $)(\mathrm{p}=0.07)$ and CsA average level was significantly higher: $232.5 \pm 106.7(\mu \mathrm{g} / \mathrm{ml})$ $(p=0.04)$. Conclusion: This study shows an increased level of CsA when it is used together with VCZ. Therapeutic drug monitoring could improve the management of the DI and optimize the co-administration of CsA and VCZ.

Key words: Drug interactions, level of cyclosporine, hematopoietic stem cell transplantation (HSCT).

Palabras clave: Interacción medicamentosa, concentraciones plasmáticas de ciclosporina, trasplante de progenitores hematopoyéticos.

\section{Introducción}

U na interacción medicamentosa (IM), "es un cambio en el modo en que actúa un medicamento cuando se administra junto con otro $u$ otros medicamentos o con alimentos. Esto puede producir alteraciones en la actividad farmacológica, es decir que el medicamento sea más o menos eficaz o que produzca efectos adversos no deseados"1, comprometiendo el objetivo del tratamiento y afectando la seguridad de administración y ser potencial de reacciones adversas a medicamentos (RAM), definida como todo efecto de un medicamento que es perjudicial y no deseado, que ocurre a dosis terapéuticas, profilácticas o de diagnóstico, según la $\mathrm{OMS}^{2}$. Los factores de riesgo para producir una IM son: edad, complejidad del tratamiento, polifarmacia, gravedad de la enfermedad, co-morbilidades y otros factores relacionados a la farmacoterapia ${ }^{3-5}$.

Numerosos estudios han mostrado una correlación entre las IMs y el número de medicamentos, principalmente en cáncer y otras condiciones que requieren tratamientos complejos, como por ejemplo el trasplante de progenitores hematopoyéticos (TPH). En diferentes etapas del TPH, los pacientes son tratados con agentes inmunosupresores, entre otros medicamentos, lo que aumenta el riesgo potencial de IM y RAM, ya que son particularmente susceptibles a IM ${ }^{3-5}$. Se observó en un estudio la frecuencia potencial de IM relacionada con el uso de un mayor número de medicamentos, siendo los antifúngicos azoles y ciclosporina los que se asocian a la ocurrencia de éstas 5 . Cuando se administran en forma conjunta ciclosporina y voriconazol, se observa un incremento del área bajo la curva de ciclosporina y una elevación de la concentración sérica de ésta, ocasionada por metabolización oxidativa, que involucra las vías: CYP2C19, CYP2C9 y CYP3A4. Asimismo, voriconazol también actúa como inhibidor de CYP3A4, haciéndolo susceptible a la interacción con ciclosporina, la cual es principalmente metabolizada por la vía del CYP3A4, ocasionando una toxicidad renal, alteraciones electrolíticas y neurotoxicidad, como RAM más agudas ${ }^{5-9}$.

La infección fúngica invasora (IFI), causada por levaduras y hongos filamentosos, es un problema persistente, con alta morbi-mortalidad entre los pacientes con 
enfermedades hematológicas y los receptores de TPH. La tasa de mortalidad media relacionada con la candidiasis invasora $(\mathrm{CI})$ es superior a $30 \%$ y mayor a $50 \%$ para aspergilosis invasora $(\mathrm{AI})^{10}$. Las características de la IFI en los pacientes onco-hematológicos están en constante evolución; mientras en los años ochenta la CI era la micosis prevalente, la generalización del uso de fluconazol profiláctico a principios de los noventa dio lugar a un gran descenso de la IFI por levaduras. Sin embargo, las IFIs por hongos filamentosos (IFI-HF) especialmente AI, han continuado siendo un importante problema médico, particularmente en portadores de: leucemias mieloblásticas agudas (LMA) y leucemias linfoblásticas agudas (LLA), síndromes mielodisplásicos de alto riesgo (SMD) y pacientes receptores de TPH alogénico (aloTPH $)^{10}$. La incidencia reportada de IFI-HF probada o probable en los pacientes hematológicos de riesgo oscila entre 4 y $22 \%$, tratándose de AI en la mayoría de los casos. Los pacientes de TPH alogénico deben recibir inmunosupresión para profilaxis de enfermedad injerto contra hospedero (EIH); el tratamiento de elección es ciclosporina. Estos pacientes tienen mayor riesgo de presentar IFI asociada a la neutropenia profunda que se induce por el acondicionamiento y tratamiento posterior al TPH. La monitorización terapéutica de estos medicamentos es realizada mediante la medición de concentraciones plasmáticas para ciclosporina y voriconazol.

\section{Objetivos}

- Describir la interacción ciclosporina-voriconazol en pacientes pediátricos hospitalizados en la Unidad de Trasplante de Médula Ósea (TMO) del Hospital Dr. Luis Calvo Mackenna.

- Generar evidencia para la toma de decisiones terapéuticas con respecto a la dosificación sugerida en presencia de esta interacción medicamentosa.

Esta IM tiene una sub-valorada notificación para el seguimiento terapéutico de estos pacientes, pudiendo ser una herramienta para la monitorización de toxicidad renal asociada a ciclosporina.

\section{Material y Método}

Estudio descriptivo, retrospectivo, que incluyó a todos los pacientes pediátricos hospitalizados entre enero de 2013 y diciembre de 2014 en la Unidad de TMO del Hospital Dr. Luis Calvo Mackenna, que recibieran voriconazol en dosis de $14 \mathrm{mg} / \mathrm{kg} / \mathrm{d}$ como tratamiento para IFI probada o probable y con uso de inmunosupresión con ciclosporina para el tratamiento y/o profilaxis de EICH. Se excluyeron los pacientes que recibieron profilaxis de EICH con otro agente anti-calcineurínico (tacrolimus,

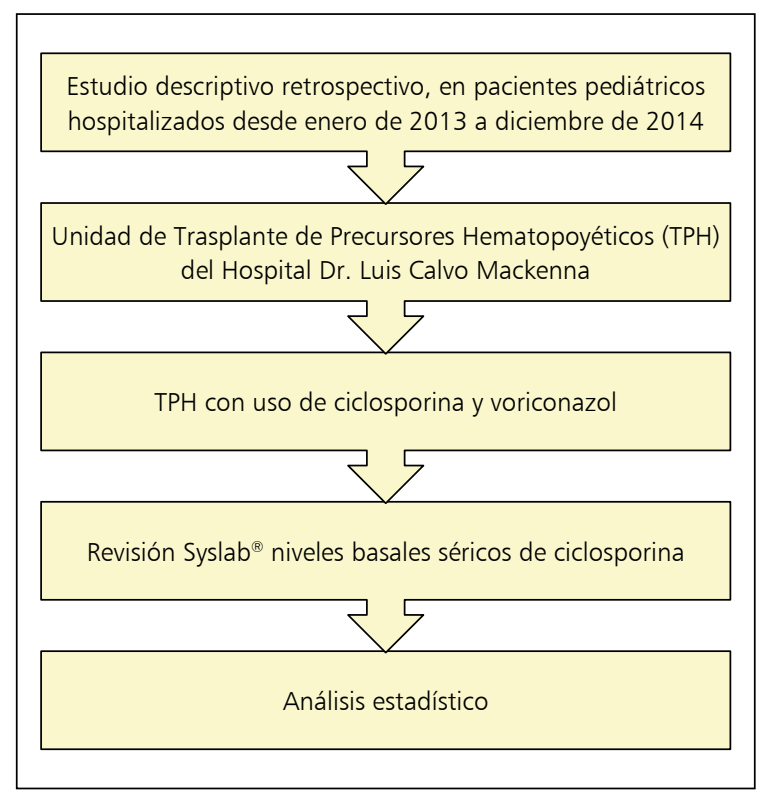

Figura 1. Esquema de la metodología.

sirolimus, etc.) y aquellos pacientes que recibieron dosis profilácticas de voriconazol. Reciben profilaxis de ciclosporina todos los pacientes receptores de aloTPH, para prevenir EICH, durante tres meses, lo que se prolonga en caso de manifestación compatible con EICH (Figura 1).

Se revisaron los resultados de las concentraciones plasmáticas basales de ciclosporina (Syslab $\left.{ }^{\circledR}\right)$, -software de laboratorio clínico para el manejo de los exámenes clínicos- de pacientes provenientes de la Unidad de TMO, muestras que fueran obtenidas por el equipo de enfermería 30 min antes de la administración de una nueva dosis del fármaco. Para la medición de las concentraciones plasmáticas se utilizó el equipo Arquitect i1000 (Abbott), que utiliza la metodología de inmunoensayo por micropartículas quimioluminiscentes (CMIA).

\section{Resultados}

Entre los años 2013 y 2014, hubo 39 episodios de pacientes receptores de TPH y oncológicos con tratamiento de voriconazol, siete de los cuales recibieron en forma concomitante ciclosporina. Dos de estos pacientes fueron excluidos por cambio del inmunosupresor. Cinco pacientes participaron en el estudio (Tabla 1). Se determinaron 63 concentraciones plasmáticas basales de ciclosporina, 27 sin voriconazol y 36 obtenidas durante el uso concomitante de voriconazol. Ninguno de estos pacientes recibió en forma concomitante medicamentos inductores del citocromo P450 que pudiesen haber contribuido a esta interacción (rifampicina ni fenitoína). 
Tabla 1. Datos demográficos de los pacientes pediátricos hospitalizados entre enero de 2013 y diciembre de 2014 con interacción ciclosporina-voriconazol

\begin{tabular}{ll}
\hline Variable & Valor \\
\hline N pacientes & 5 \\
\hline Diagnóstico de base & TPH-alogénico \\
\hline Edad (años) [rango] & $5[3-7]$ \\
\hline Sexo masculino (\%) & $5(100)$ \\
\hline Peso (kg) [rango] & $20[17-30]$ \\
\hline N de muestras (episodios) & 39 \\
\hline
\end{tabular}

Tabla 2. Número de muestras de concentraciones plasmáticas basales de ciclosporina tomadas para cada paciente pre y post uso de tratamiento con voriconazol

\begin{tabular}{ccc}
\hline n paciente & $\begin{array}{c}\text { Muestras plasmáticas basales de ciclosporina } \\
\text { Durante el uso de voriconazol } \\
\text { (n: } \mathbf{3 6})\end{array}$ \\
\hline 1 & $\begin{array}{c}\text { Previo al uso de voriconazol } \\
\text { (n: } \mathbf{2 7} \text { ) }\end{array}$ & 6 \\
\hline 2 & 6 & 4 \\
\hline 3 & 6 & 9 \\
\hline 4 & 5 & 10 \\
\hline 5 & 5 & 7 \\
\hline Total & 5 & 36 \\
\hline
\end{tabular}

Tabla 3. Revisión de dosificación y concentraciones plasmáticas de ciclosporina de los pacientes que reciben profilaxis de EICH en conjunto con voriconazol pre y post tratamiento

\begin{tabular}{|c|c|c|c|}
\hline & $\begin{array}{l}\text { Previo al uso de voriconazol } \\
\text { (n:27) }\end{array}$ & $\begin{array}{l}\text { Durante el uso de voriconazol } \\
\qquad(\mathrm{n}: 36)\end{array}$ & Valor $p$ \\
\hline Dosis de ciclosporina (mg/kg/día) & $4,6 \pm 2,6$ & $5,5 \pm 3,0$ & $0,0709^{\mathrm{a}}$ \\
\hline Vía de administración (\% de mediciones con vía endovenosa) & 51,9 & 38,9 & $0,2211^{b}$ \\
\hline C. plasmática de ciclosporina ( $\mu \mathrm{g} / \mathrm{ml})$ & $188,8 \pm 84,1$ & $232,5 \pm 106,7$ & $0,042^{\mathrm{a}}$ \\
\hline
\end{tabular}

Datos cuantitativos presentados como promedio \pm desviación estándar; ${ }^{a T e s t ~ d e ~ l a ~ t ~ d e ~ S t u d e n t ; ~}{ }^{b}$ Prueba exacta de Fischer.

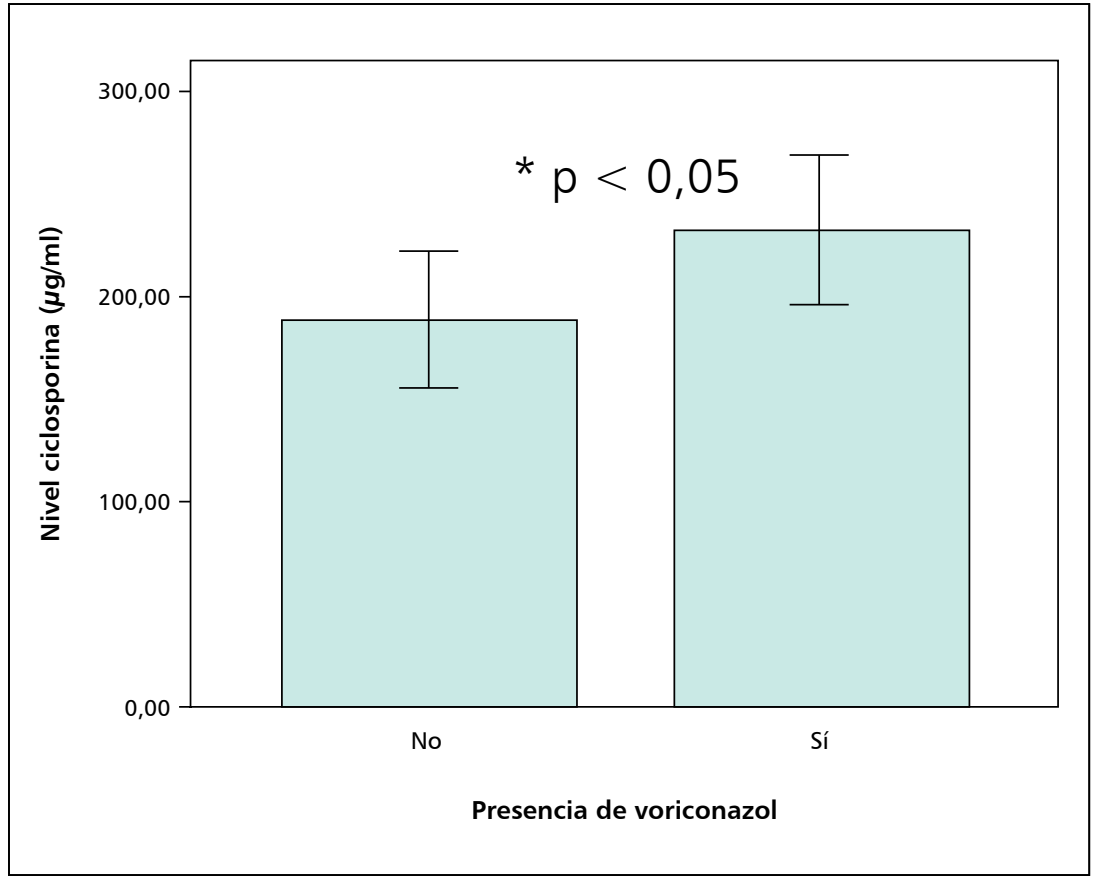

Figura 2. Promedio de las concentraciones plasmáticas de ciclosporina previas a la administración de voriconazol y en administración conjunta.
El detalle de las concentraciones plasmáticas de ciclosporina basal tomados en cada paciente se puede ver en la Tabla 2.

No se observó diferencia significativa en la dosis de ciclosporina y la proporción de uso de vía endovenosa entre los grupos con y sin voriconazol. Se observó diferencia significativa en las concentraciones plasmáticas de ciclosporina alcanzadas para ambos grupos. Las características de las variables se pueden apreciar en la Tabla 3.

En la Figura 2 se observan las concentraciones plasmáticas basales promedio de ciclosporina previas a la administración de voriconazol, con un incremento de $23,1 \%$ en la concentración tras la administración del antifúngico.

\section{Discusión}

Los resultados encontrados mostraron una concordancia con lo ya descrito en la literatura científica. A. H. Groll y cols., describieron un paciente pediátrico con TPH en terapia concomitante con voriconazol, donde se incrementaban las concentraciones plasmáticas de ciclosporina en 1,7 veces en el área bajo la curva (ABC). Ese estudio 
fue diseñado para examinar el efecto de voriconazol en la farmacocinética de ciclosporina y en la seguridad de la administración concomitante y demuestra la interacción farmacológica entre ambos ${ }^{8}$. En nuestra revisión realizada en pacientes pediátricos, encontramos similitud respecto al incremento inicial de las concentraciones plasmáticas de ciclosporina junto a la administración concomitante de voriconazol, observándose un aumento de $23,1 \%$ en las concentraciones plasmáticas de ciclosporina.

Los resultados encontrados en estos pacientes, podrían entregar antecedentes para la determinación de RAM asociadas a ciclosporina por altas concentraciones, especialmente a nivel renal (incremento de creatininemia, insuficiencia renal aguda, hiperkalemia), generando una disminución en los riesgos de deterioro de función renal asociados a la terapia inmunosupresora que deben recibir los pacientes. La farmacovigilancia asociada para individualizar las dosis de ciclosporina en los pacientes con voriconazol nos puede ayudar con esta cohorte de pacientes para generar un ajuste de dosis $20 \%$ menor de la dosis total de ciclosporina, en paciente que iniciaran tratamiento con voriconazol. Este ajuste se asocia a $23,1 \%$ de incremento de la concentración plasmática de ciclosporina en presencia de voriconazol.

Este estudio aporta datos preliminares; limitantes manifiestas son el tamaño de muestra pequeño y un diseño retrospectivo lo que nos motiva a ampliar esta vigilancia y estudios a futuro. Otras limitantes importantes fueron que:

- Se debió hacer un seguimiento mayor en el tiempo para saber si existen RAM.

- Faltaron más monitorizaciones de otros medicamentos que pudieran interferir con la excreción de ciclosporina.

- Además, no hubo evaluación del impacto clínico de la interacción farmacológica; sólo se describió la interacción farmacocinética, no la implicancia clínica.

\section{Conclusión}

Esta experiencia ratifica la importancia de la monitorización de interacción de voriconazol y ciclosporina en los pacientes de TPH. Nuestra sugerencia para estos pacientes es disminuir en $20 \%$ la dosis de ciclosporina al inicio de una terapia concomitante con voriconazol y controlar las concentraciones plasmáticas de ciclosporina luego de $72 \mathrm{~h}$ o a la $5^{\circ}$ vida media de inicio del tratamiento, ya sea cuando los pacientes sean expuestos a terapias de ciclosporina o voriconazol, para evitar dosis sub-terapéuticas o sobredosis que pudiesen traducirse en interacciones medicamentosas y potenciales RAM que pusieran en riesgo la seguridad de nuestros pacientes.

Agradecimientos. A los miembros de la Unidad de Trasplante de Medula Ósea, Laboratorio y Unidad de Farmacia, Hospital Dr. Luis Calvo Mackenna. Además, a la Unidad de Investigación, Facultad de Medicina, Universidad de Chile, Hospital Dr. Luis Calvo Mackenna.

\section{Resumen}

Introducción: Las interacciones medicamentosas (IM) en el trasplante de progenitores hematopoyéticos (TPH) son comunes y clínicamente significativas, especialmente en: anticonvulsivantes, voriconazol (VCZ) y ciclosporina (CsA). Objetivo: Describir las interacciones de CsA-VCZ en pacientes con TPH. Métodos: Estudio descriptivo, retrospectivo, en pacientes receptores de TPH entre enero de 2013 y diciembre de 2014 en la Unidad de Trasplante de Médula Ósea del Hospital Dr. Luis Calvo Mackenna, que recibieran CsA y VCZ. Resultados: Edad media: 5 años (3-6), peso promedio: $20 \mathrm{~kg}$ (17-30). Se analizaron 63 concentraciones plasmáticas de CsA, 27 eran concentraciones de CsA previas al uso de VCZ y 36 concentraciones plasmáticas de CsA concomitantes con VCZ. En el grupo de CsA previo a VCZ, la dosis de CsA fue 4,6 $\pm 2,6(\mathrm{mg} / \mathrm{kg} /$ día $)$ y la concentración media de CsA $188,8 \pm 84,1(\mu \mathrm{g} / \mathrm{ml})$. En el grupo de CsA en forma concomitante con VCZ, la dosis de CsA fue de 5,5 $\pm 3,0$ (mg/kg/día) (p 0,07) y la concentración media de CsA fue: $232,5 \pm 106,7(\mu \mathrm{g} / \mathrm{ml})(\mathrm{p}=0,04)$. Conclusión: Se demostró un aumento de las concentraciones plasmáticas de CsA en IM con VCZ. La monitorización terapéutica podría mejorar el manejo de la IM y optimizar la coadministración de CsA y VCZ.

\section{Referencias bibliográficas}

1.- Uso racional de medicamentos: Una tarea de todos. Dpto. Políticas Farmacéuticas y Profesiones Médicas División de Políticas Públicas saludables y Promoción Subsecretaría de Salud Pública 2012. http://web.minsal.cl/ portal/url/item/8da19e5eac7b8164e0400101 1e012993.pdf
2.- Morales M, Ruiza I, Morgado C, González X. Farmacovigilancia en Chile y el mundo. Rev Chilena Infectol 2002; 19: Supl. 1; http://dx.doi.org/10.4067/S0716-10182002019 100008.

3.- Mazzei T. The difficulties of polytherapy: examples from antimicrobial chemotherapy. Intern Emerg Med 2011; 6 Suppl 1: S103-S109.
4.- Guastaldi R, Moreira A, Figueras A, Regina S. Prevalence of potential drug-drug interactions in bone marrow transplant patients. Int J Clin Pharm 2011; 33: 1002-9.

5.- Fernández de Palencia Espinosa MA, Díaz Carrasco MS, Fuster Soler JL, Ruíz Merino G, De la Rubia Nieto MA, Espuny Miró A. Pharmacoepidemiological study of drug-drug interactions in onco- 
hematological pediatric patients. Int J Clin Pharm 2014; 36 (6): 1160-9.

6.- Bernard E, Goutelle S, Bertrand Y, Bleyzac N. Pharmacokinetic drug-drug interaction of calcium channel blockers with cyclosporine in hematopoietic stem cell transplant children. Ann Pharmacother 2014; 48 (12): 1580-4.

7.- Hegazy S, Adam A, Hamdy N, Khalafallah N. Effect of active infection on cytochrome P450- mediated metabolism of cyclosporine in renal transplant patients. Transplant Infect Dis 2015; 17 (3): 350-60.

8.- Groll A, Kolve H, Ehlert K, Paulussen M, Vormoor J. Pharmacokinetic interaction between voriconazole and cyclosporine A following allogeneic bone marrow transplantation. J Antimicrob Chemother 2004; 53: 113-4.
9.- Romero A J, Le Pogamp P, Nilsson L G. Wood N. Effect of voriconazole on the pharmacokinetics of cyclosporine in renal transplant patients. Clin Pharmacol Ther 2002; 71: 226-34

10.- Vallejo J C, Ruiz-Camps I. Infección fúngica invasora en los pacientes hematológicos. Enferm Infecc Microbiol Clin 2012; 30 (9): 572-9. 